\title{
Potential inhibitors targeting RNA-dependent RNA polymerase activity (NSP12) of SARS-CoV-2
}

Zijing Ruan ${ }^{1,2}$, Chao Liu ${ }^{1}$, Yuting Guo ${ }^{1}$, Zhenqing $\mathrm{He}^{1}$, Xinhe Huang ${ }^{2}$, Xu Jia ${ }^{1}$, Tai Yang $^{3}$

1 Non-coding RNA and Drug Discovery Key Laboratory of Sichuan Province, Chengdu Medical College, Chengdu, Sichuan, China

2 School of Life Science and Engineering, Southwest Jiaotong University, Chengdu, Sichuan, China

3 School of Pharmacy, Chengdu Medical College, Chengdu, Sichuan, China

* Correspondence to: Tai Yang: taiyang@cmc.edu.cn

Xu Jia: jiaxu@cmc.edu.cn

Xinhe Huang: xinhehuang@swjtu.edu.cn 


\begin{abstract}
A novel coronavirus (SARS-CoV-2) that is initially found to trigger human severe respiratory illness in Wuhan City of China in 2019, has killed 2,718 people in China by February 26, 2020, and which has been recognized as a public health emergency of international concern as well. And the virus has spread to more than 38 countries around the world. However, the drug has not yet been officially licensed or approved to treat SARS-Cov-2 infection. NSP12-NSP7-NSP8 complex of SARS-CoV-2, essential for viral replication and transcription, is generally regarded as a potential target to fight against the virus. According to the NSP12-NSP7-NSP8 complex (PDB ID: 6NUR) structure of SARS, two homologous models were established for virtual screening in present study, namely NSP12-NSP7 interface model and NSP12-NSP8 interface model. Seven compounds (Saquinavir, Tipranavir, Lonafarnib, Tegobuvir, Olysio, Filibuvir and Cepharanthine) were selected for binding free energy calculations based on virtual screening and docking scores. All the seven compounds can combine well with NSP12NSP7-NSP8 in the homologous model, providing drug candidates for the treatment and prevention of SARS-CoV-2.
\end{abstract}

Keywords: SARS-CoV-2; Nonstructural proteins (NSP); NSP12; NSP7; NSP8; Virtual screening; Inhibitor 


\section{Introduction}

Since the outbreak of pneumonia caused by a novel coronavirus (SARS-CoV-2) in Wuhan, China in December 2019, the world have faced unprecedented challenges in treating the disease caused by this virus [1-3]. China has implemented strict epidemic prevention and control measures across the country to avoid larger scale epidemic [4]. However, as of February 26, 2020, there were 78,195 confirmed cases and more than 2,718 deaths. The virus now presents in more than 30 countries. The infectious power and harmful effects of SARS-CoV-2 should not be underestimated. To date, there are no officially licensed or approved drugs against this novel coronavirus. There is an urgent need to find new targets for development of anti-SARS-CoV-2 agents.

ORF1a and ORF1b at the 5'-terminus of the Coronavirus $(\mathrm{CoV})$ genome encode polyprotein $1 \mathrm{a}$ and polyprotein $1 \mathrm{~b}$, the two proteins could be cleaved into 16 nonstructural proteins (NSPs), which are essential for viral replication and transcription, thus being regarded as a potential virulence factor and a target for $\mathrm{CoV}[5,6]$. Among these NSPs, the NSP12 subunit is the essential RdRp (rna-dependent RNA polymerase) of the coronavirus replicative machinery, which was even able to extend a homopolymeric primer-template substrate by a few dozen nucleotides in vitro [7]. The $3.1 \AA$ cryo-EM structure of the SARS-CoV RNA polymerase NSP12 shows it can bind with its essential co-factors NSP7 and NSP8 [8]. The replication of the SARScoronavirus genome involves two RNA-dependent RdRps. The first is primerdependent and associated with the NSP12, whereas the second is catalyzed by NSP8. NSP8 is capable of de novo initiating replication process and has been proposed to operate as a primase [9]. In addition, NSP7, a component of the CoV replicase polyprotein, also participates in viral replication processed by binding to NSP12 as another primase [9]. The NSP12 needs to associate with NSP7 and NSP8 to activate its capability to replicate long RNA [7]. This elicit us to identify the particularly interesting compound disrupt the binding of NSP7 or NSP8 to NSP12, thus which could be used to inhibit the RdRp activity of NSP12, acting as novel antiviral agents and therapies of 


\section{SARS-Cov-2.}

The amino acid sequence alignment revealed that the NSP12 of SARS-CoV-2 shared 96.35\% similarity with the NSP12 (PDB ID: 6NUR) of SARS (Figure 1). In addition, comparative analyses of their deduced amino acid sequences revealed that NSP7 and NSP8 of SARS-CoV-2 shared $98.8 \%$ and $97.5 \%$ similarity with that of SARS-CoV respectively. Therefore, the SARS-CoV-2 NSP12 structures could be constructed by the performance of homology modeling using SARS NSP12 (PDB ID: 6NUR) as template. The NSP7 and NSP8 binding pocket of NSP12 were designated as active sites for screening compounds. Here, through high-throughput screening methods using a pool of 30,000 small molecules, several potential drug candidates were identified for preventing the binding NSP7 or NSP8 to NSP12, suggesting further assessment of the anti-SARS-CoV-2 activity of these compounds in cell culture.

\section{Materials and Methods}

\subsection{Construction of small molecular ligands}

Each sub-library (FDA, world-not-FDA, investigational-only, http://zinc.docking.org/substances/subsets/) was downloaded from the zinc database. Then the model was converted to pdbqt format by prepare_receptor4.py script with assigning atomic types and atomic charges. All rotatable bonds in the molecule are set to be flexible for flexible docking.

\subsection{Preparation of target proteins}

Homology model of the target protein (NSP12, NSP7 and NSP8 of SARS-CoV-2) was built by modeller 9.18 using crystal structure of SARS NSP12 (PDB ID: 6NUR) as template. 100 independent structures were constructed and the one with best DOPE score was chosen for further energy minimization by Amber. The relaxed model was saved as pdb file, and which was converted to pdbqt format as docking receptor using AutoDockTools-1.5.6, with assigned atom type and partial charge. 


\subsection{Molecular docking}

Vina1.1.2 was used to perform molecular docking. The docking boxes were set at the NSP12-NSP7 interface and NSP12-NSP8 interface, respectively. The search exhaustiveness was set to be 32, and the number of binding modes was set to be 9 . Other parameters were set as default. During docking, NSP7 (or NSP8) was removed from the complex and only NSP12 was left as receptor.

\subsection{Binding free energy calculation}

Each simulation system was immersed in a cubic box of TIP3P water with $10 \AA$ distance from the solute. The $\mathrm{Na}^{+}$or $\mathrm{Cl}^{-}$was applied to neutralize the system. General Amber force field (GAFF) 15 and Amber ff14SB force field were used to parameterize the ligand and protein respectively. 10,000 steps of minimization with constraints (10 $\mathrm{kcal} / \mathrm{mol} / \AA \AA 2$ ) on heavy atoms of complex, including 5,000 steps of steepest descent minimization and 5,000 steps of conjugate gradient minimization, was used to optimize each system. Then each system was heated to $300 \mathrm{~K}$ within $0.2 \mathrm{~ns}$ followed by $0.1 \mathrm{~ns}$ equilibration in NPT ensemble. Finally, 5 ns MD simulation on each system at $300 \mathrm{~K}$ was performed. The minimization, heating and equilibrium are performed with sander program in Amber18. The 5 ns production run was performed with pmemd.cuda. Based on the $5 \mathrm{~ns}$ MD simulation trajectory, binding free energy $(\Delta \mathrm{G})$ was calculated with MM/GBSA method according to the following equation: $\Delta \mathrm{G}_{\mathrm{cal}}=\Delta H-$ $\mathrm{T} \Delta \mathrm{S}=\Delta \mathrm{E}_{\mathrm{vdw}}+\Delta \mathrm{E}_{\text {ele }}+\Delta \mathrm{G}_{\mathrm{gb}}+\Delta \mathrm{G}_{\mathrm{np}}-\mathrm{T} \Delta \mathrm{S}$, where $\Delta \mathrm{E}_{\text {ele }}$ and $\Delta \mathrm{EVDW}$ refer to electrostatic and van der Waals energy terms respectively. $\Delta \mathrm{G}_{\mathrm{gb}}$ and $\Delta \mathrm{G}_{\mathrm{np}}$ refer to polar and non-polar solvation free energies respectively. Conformational entropy $(\mathrm{T} \Delta \mathrm{S})$ was not calculated for saving time. Besides, the ligands were compared based on the same target, so it is reasonable to ignore the entropy.

\section{Results and discussion}

\subsection{Docking results of 7496 drugs against NSP12-NSP7 model and NSP12-NSP8 model}


The 7,964 drugs obtained from the zinc database were screened for molecular docking. Among them, 151 of the 2,100 compounds approved by the FDA have a docking score better than $-7.0 \mathrm{kcal} / \mathrm{mol}$. There were 4,264 compounds approved by regulatory agencies other than the FDA. Among them, there were 295 compounds with the docking score better than $-7.0 \mathrm{kcal} / \mathrm{mol}$. 134 of the 1132 compounds in clinical trials have a docking score better than $-7.0 \mathrm{kcal} / \mathrm{mol}$. Further, thirteen top compounds showed the docking score in a range of -7.5 to $-8.4 \mathrm{kcal} / \mathrm{mol}$ were selected from docking results from the NSP12-NSP7 interface of homology model (Table 1). Similarly, eleven top compounds showed the docking score in a range of -7.7 to $-8.4 \mathrm{kcal} / \mathrm{mol}$ were selected from the NSP12-NSP8 interface of homology model (Table 2). Among the selected drugs, Cepharanthine and Tegobuvir can block both the NSP12-NSP7 interface and the NSP12-NSP8 interface. Further, Olysio, Filibuvir, Tipranavir, Saquinavir and Lonafarnib were all closely associated with antiviral activity.

Table 1 Thirteen compounds selected from the NSP12-NSP7 interface of homology model

Table 1 Thirteen compounds selected from the NSP12-NSP7 interface of homology model

\begin{tabular}{cccc} 
Compounds name & ID & Data & Affinity (kcal/mol) \\
\hline Lonafarnib & ZINC3950115 & Investigational-only & -8.4 \\
Tegobuvir & ZINC100057121 & Investigational-only & -8.2 \\
Aromasin & ZINC3973334 & FDA & -8 \\
Troglitazone & ZINC968279 & World-not- FDA & -8 \\
Hypericin & ZINC3780340 & Investigational-only & -8 \\
Nilotinib & ZINC6716957 & FDA & -7.9 \\
Cepharanthine & ZINC30726863 & World-not- FDA & -7.9 \\
Eltrombopag & ZINC11679756 & FDA & -7.8 \\
Tipranavir & ZINC100016058 & FDA & -7.8 \\
Saquinavir & ZINC26985532 & FDA & -7.8 \\
Indinavir & ZINC22448696 & FDA & -7.7 \\
Cobicistat & ZINC85537014 & FDA & -7.7 \\
Talmapimod & ZINC34001955 & Investigational-only & -7.5 \\
\hline
\end{tabular}


Table 2 Eleven compounds selected from the NSP12-NSP8 interface of homology model

\section{Table 2 Eleven compounds selected from the NSP12-NSP8 interface of homology model}

\begin{tabular}{cccc} 
Compounds name & ID & Data & Affinity (kcal/mol) \\
\hline Nilotinib & ZINC6716957 & FDA & -8.4 \\
Tegobuvir & ZINC100057121 & Investigational-only & -8.4 \\
Olysio & ZINC164760756 & FDA & -8.3 \\
Cepharanthine & ZINC30726863 & World-not- FDA & -8.3 \\
Rimegepant & ZINC68267814 & Investigational-only & -8.2 \\
Eltrombopag & ZINC11679756 & FDA & -8.1 \\
Biosone & ZINC19203131 & World-not- FDA & -7.9 \\
Hypericin & ZINC3780340 & Investigational-only & -7.9 \\
Lurasidone & ZINC3927822 & FDA & -7.8 \\
Avodart & ZINC3932831 & FDA & -7.8 \\
Filibuvir & ZINC100078465 & Investigational-only & -7.7 \\
\hline
\end{tabular}

\subsection{Docking results of Saquinavir and Tipranavir against SARS-CoV-2 NSP12- NSP7 model}

Saquinavir, the first HIV protease inhibitor was introduced into the market in 1995. Tipranavir, a novel non-peptide protease inhibitor approved for use in patients with resistant strains of HIV. Both of which are safe and generally well-tolerated in HIV-1infected adults $[10,11]$. Our docking results showed that five of the hydrogen bonds involving GLY-297, PHE-299, PHE-325, PHE-326 and ALA-327 maintained upon the binding of saquinavir with interface between SARS-CoV-2 NSP12 and NSP7 (Figure 1A). As for Tipranavir, hydrogen bonds involving PHE-325 maintained upon the binding of Tipranavir with interface between SARS-CoV-2 NSP12 and NSP7 (Figure 1C). Saquinavir and Tipranavir could bind to the interface active pockets of the SARSCoV-2 NSP12 and NSP7 (Figure 1B, D). The previous study showed that saquinavir could bind to the SARS-CoV-2 RNA-dependent RNA polymerase and inhibit the enzyme activity[12]. Our observations further confirm that saquinavir and tipranavir can bind to the NSP12-NSP7 interface as interfacial blockers, thus making them as candidates for further in vitro evaluation of anti-SARS-CoV-2 activity. 

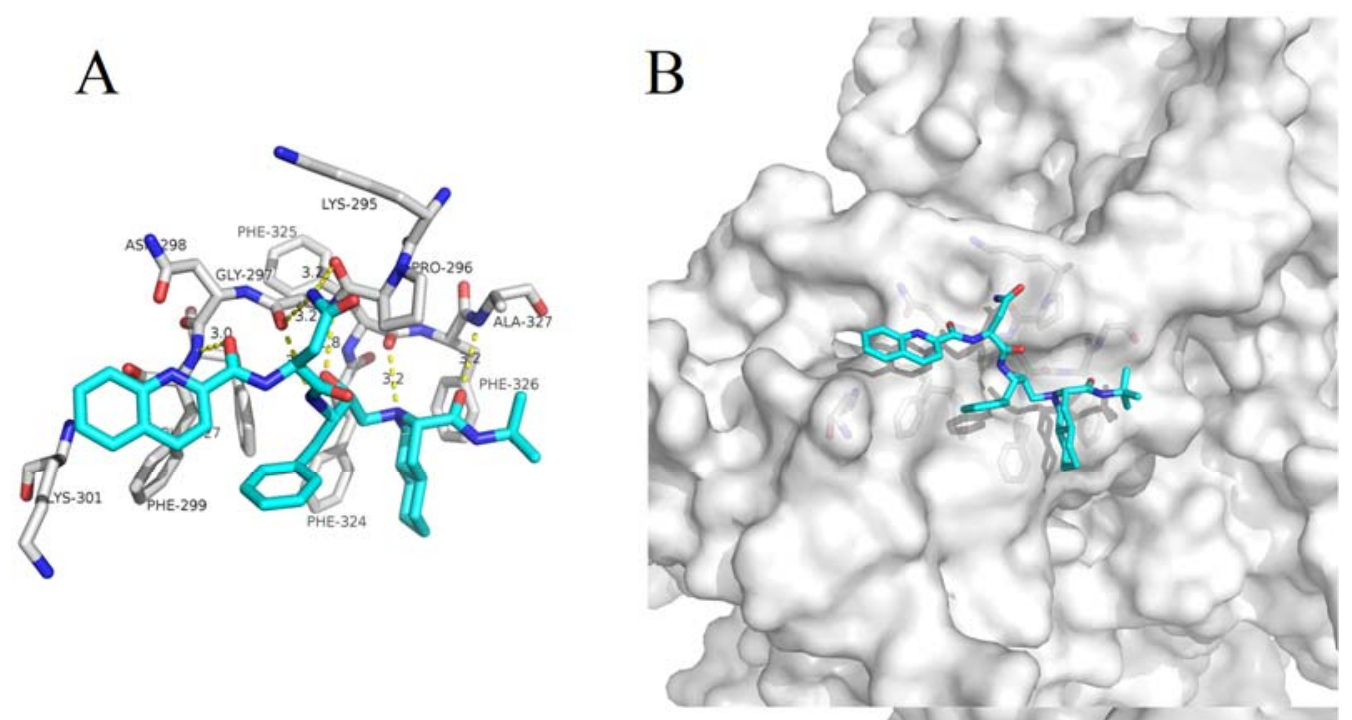

C

$\mathrm{D}$
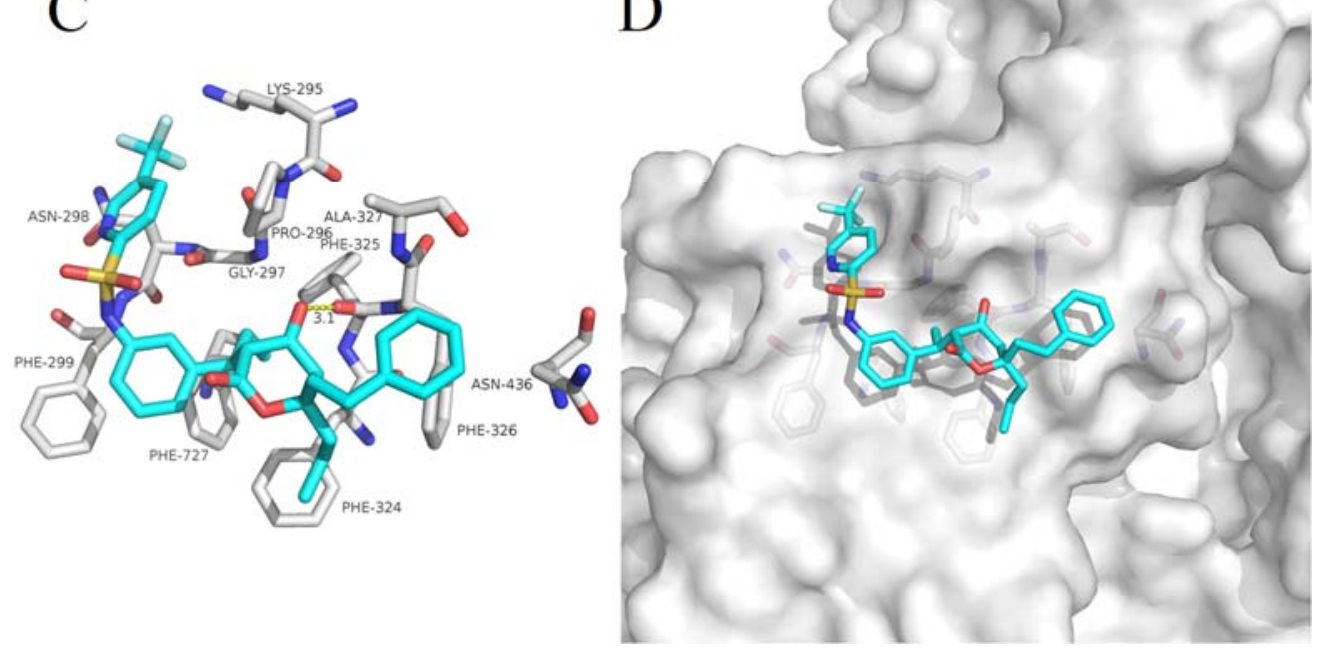

Figure 1. The binding model of Saquinavir and Tipranavir against SARS-CoV-2 NSP12-NSP7. (A) Interactions between Saquinavir (cyan) and associated residues (off-white) in the interface of the homology model for SARS-CoV-2; (B) Binding models of Saquinavir (cyan) in the SARS-CoV-2 NSP12-NSP7 protein interface pocket (white surface); (C) Interactions between Tipranavir (cyan) and associated residues (off-white) in the interface of the homology model for SARS-CoV-2; (D) Binding models of Tipranavir (cyan) in the SARS-CoV-2 NSP12-NSP7 protein interface pocket (white surface). Numbers accompanying dashed yellow lines represents the interaction distance $(\AA)$.

\subsection{Docking results of Lonafarnib against SARS-CoV-2 NSP12-NSP7 model}

Lonafarnib as a non-peptidomimetic inhibitor of farnesyltransferase has been used for progeria $[13,14]$. Our docking results showed that lonafarnib was mainly combined with the interface between SARS-CoV-2 NSP12 and NSP7 through van der Waals potential energy and hydrophobic accumulation, involving PHE-299, PHE-727, PHE- 
324, PHE-325 and PHE-326 (Figure 2A). Lonafarnib could bind to the interface active pockets between the SARS-CoV-2 NSP12 and NSP7 (Figure 2B). Therefore, we speculate that Lonafarnib has potential activity for the treatment of SARS-Cov-2 infection.
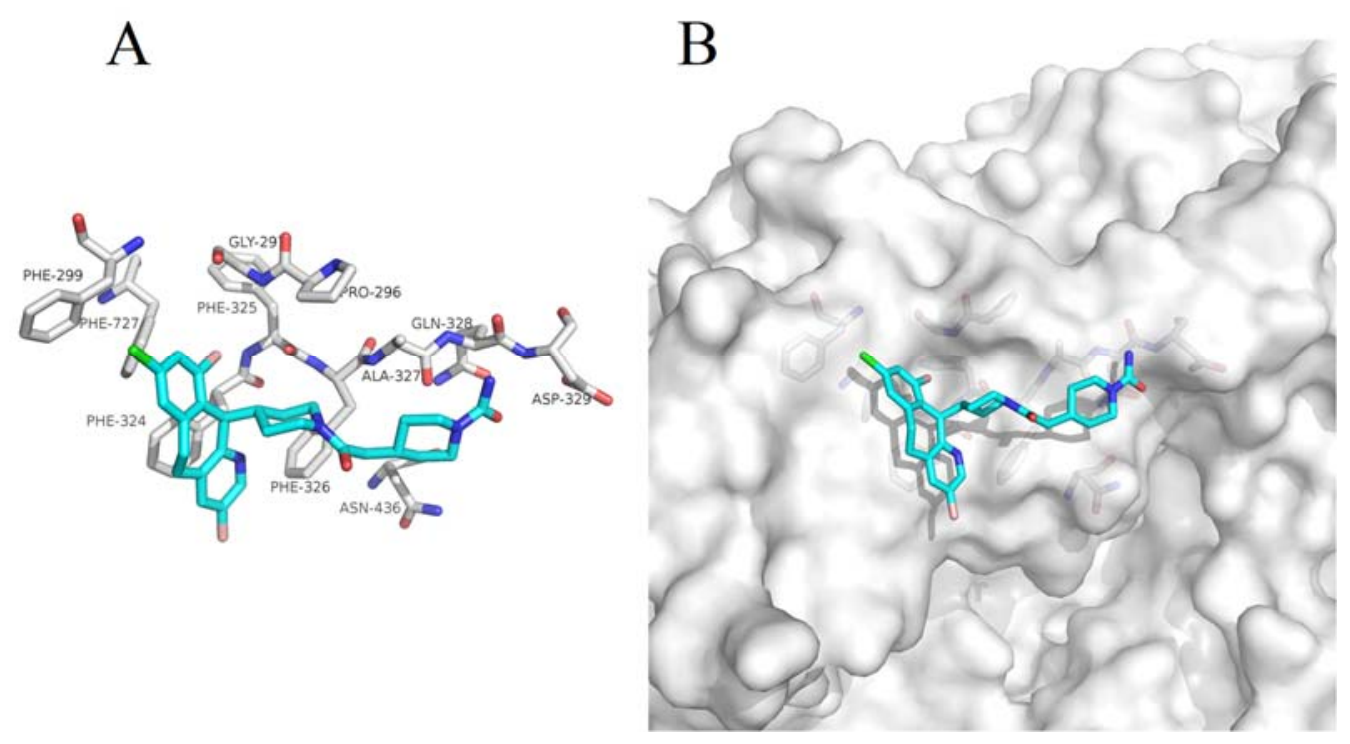

Figure 2. The binding model of Lonafarnib against SARS-CoV-2 NSP12-NSP7. (A) Interactions between Lonafarnib (cyan) and associated residues (off-white) in the interface of the homology model for SARS-CoV-2; (B) Binding models of Lonafarnib (cyan) in the SARS-CoV-2 NSP12NSP7 protein interface pocket (white surface).

\subsection{Docking results of Tegobuvir against SARS-CoV-2 NSP12-NSP7 model and NSP12-NSP8 model}

Tegobuvir (GS-9190), a novel imidazopyridine inhibitor of hepatitis C virus (HCV) RNA replication in vitro and has demonstrated potential antiviral activity in patients chronically infected with genotype $1 \operatorname{HCV}[15,16]$. Our docking results showed that Tegobuvir was mainly combined with the interface between SARS-CoV-2 NSP12 and NSP7 through van der Waals potential energy and hydrophobic accumulation, involving PHE-299, PHE-727, PHE-324, PHE-325 and PHE-326 (Figure 3A). Further, van der Waals forces mainly maintain the bond between Tegobuvir and the SARS-CoV2 NSP12-NSP8 interface (Figure 3C). Moreover, Tegobuvir could also bind to both of 
the interface active pockets of the SARS-CoV-2 NSP12-NSP7 and NSP12-NSP8

(Figure 3B, D). Thus, Tegobuvir could be as a candidate drug against SARS-CoV-2.

A

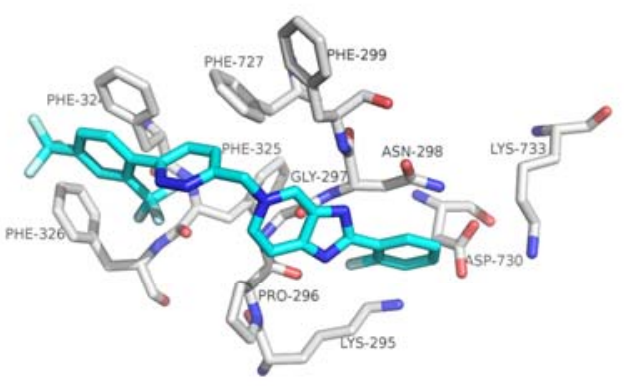

$\mathrm{C}$

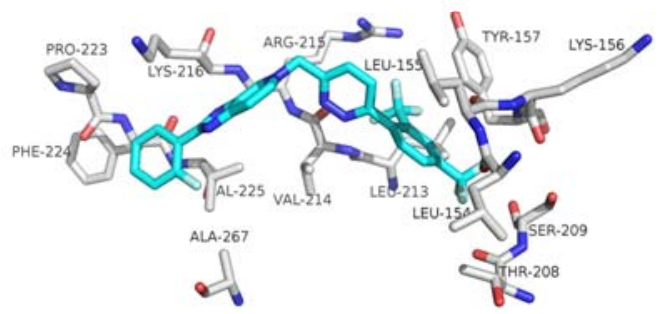

B

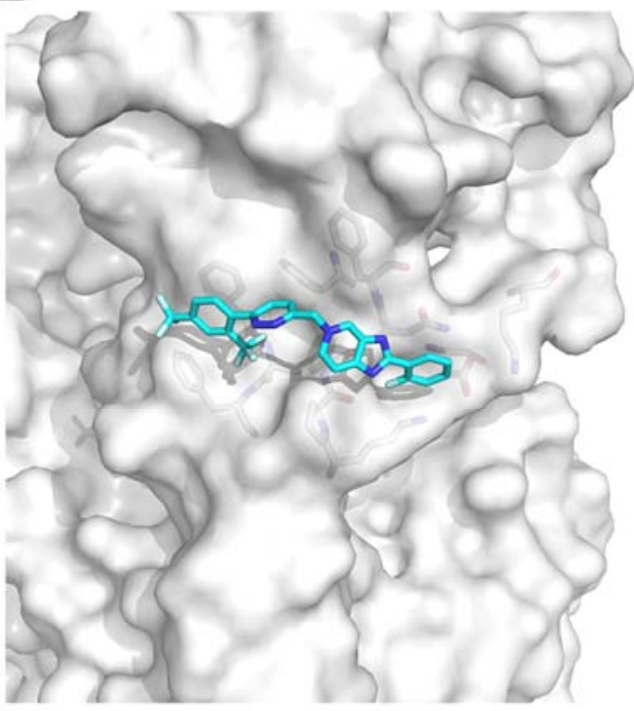

$\mathrm{D}$

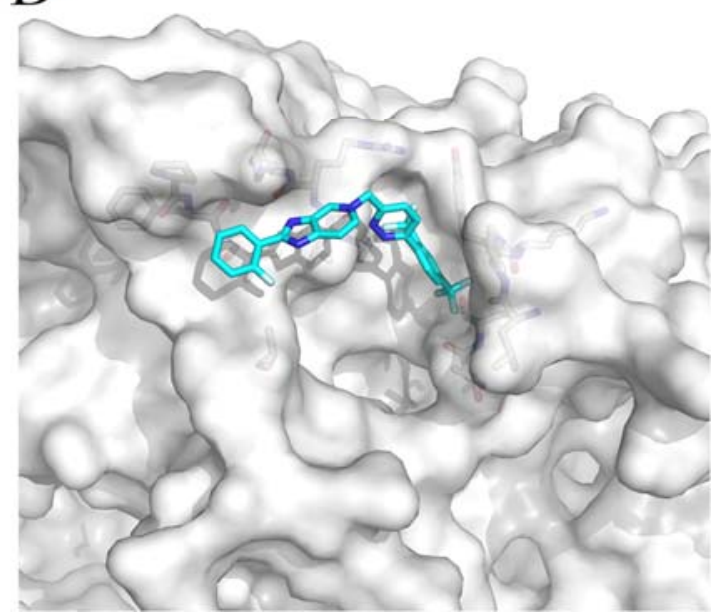

Figure 3. The binding model of Tegobuvir against SARS-CoV-2 NSP12-NSP7 and SARS-CoV-2 NSP12-NSP8. (A) Interactions between Tegobuvir (cyan) and associated residues (off-white) in the interface of the homology model for SARS-CoV-2; (B) Binding models of Tegobuvir (cyan) in the SARS-CoV-2 NSP12-NSP7 protein interface pocket (white surface);(C) Interactions between Tegobuvir (cyan) and associated residues (off-white) in the interface of the homology model for SARS-CoV-2; (D) Binding models of Tegobuvir (cyan) in the SARS-CoV-2 NSP12-NSP8 protein interface pocket (white surface).

\subsection{Docking results of Olysio against SARS-CoV-2 NSP12-NSP8 model}

Olysio, a hepatitis c virus (HCV) NS3/4A protease inhibitor approved for the 
treatment of genotype 1 chronic hepatitis $\mathrm{C}$ in combination with pegylated interferon and ribavirin [17]. Our docking results showed that the hydrogen bonds involving VAL214 and van der Waals forces maintained upon the binding of Olysio and SARS-CoV2 NSP12-NSP8 interface (Figure 4A). Olysio could also bind to the interface active pockets of the SARS-CoV-2 NSP12-NSP8 (Figure 4B). Thus, based on the present results, Olysio may be considered as a candidate for further in vitro evaluation of antiSARS-CoV-2 activity.
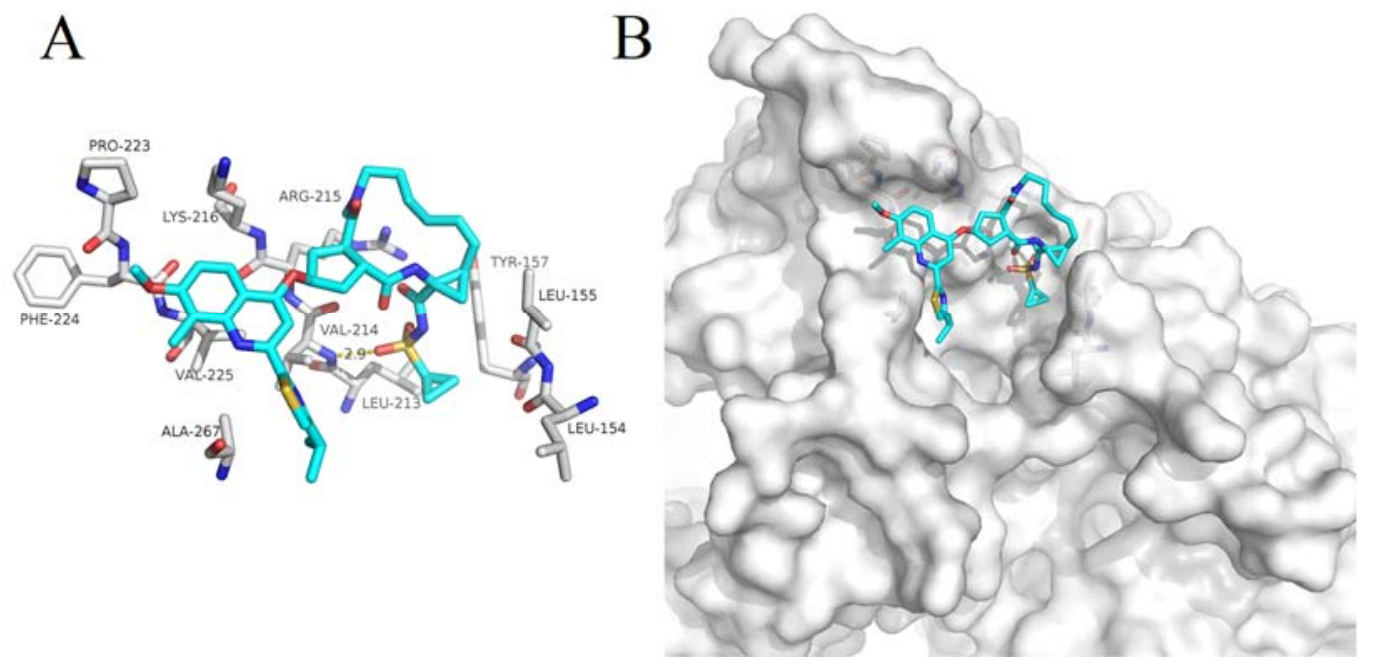

Figure 4. The binding model of Olysio against SARS-CoV-2 NSP12-NSP8. (A) Interactions between Olysio (cyan) and associated residues (off-white) in the interface of the homology model for SARS-CoV-2; (B) Binding models of Olysio (cyan) in the SARS-CoV-2 NSP12-NSP8 protein interface pocket (white surface). Numbers accompanying dashed yellow lines represents the interaction distance $(\AA)$.

\subsection{Docking results of Cepharanthine against SARS-CoV-2 NSP12-NSP8 model}

Cepharanthine, an alkaloid tetrandrine isolated from Stephania tetrandra was found to exert strong anti-cancer, anti-inflammatory and antioxidant activities [18]. In addition, it shows in vitro inhibitory effect on Herpes simplex virus type 1 (HSV-1) infected cells [19]. Our docking results showed that the hydrogen bonds involving ARG-215 maintained upon the binding of Cepharanthine and SARS-CoV-2 NSP12NSP8 interface, with additionally van der Waals forces (Figure 5A). Cepharanthine could bind to the interface active pockets of the SARS-CoV-2 NSP12-NSP8 (Figure 
5B). The previous study showed Cepharanthine could significantly inhibit the replication of human coronavirus strains OC43 [18]. Taken together, Cepharanthine coule be a potential natural antiviral compound for the prevention and treatment of SARS-CoV-2 infection.

A

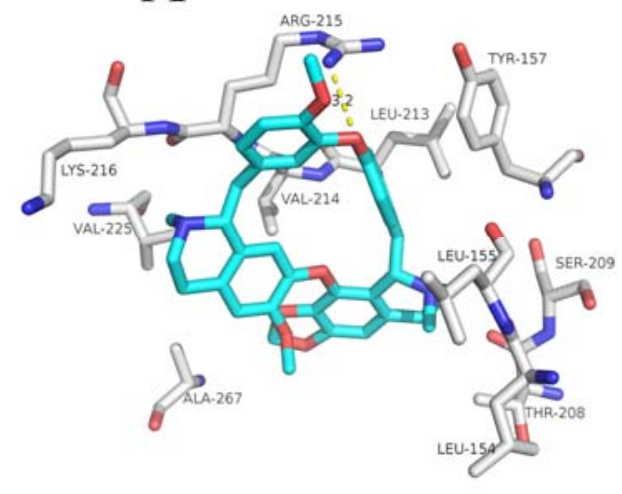

$\mathrm{B}$

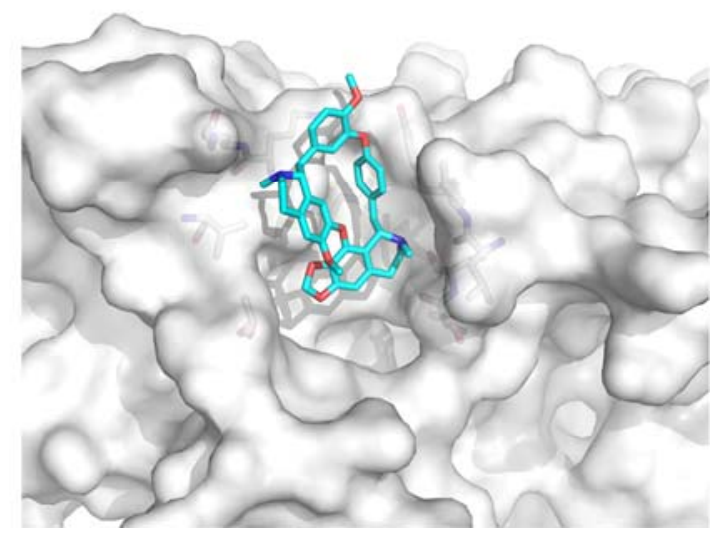

Figure 5. The binding model of Cepharanthine against SARS-CoV-2 NSP12-NSP8. (A) Interactions between Cepharanthine (cyan) and associated residues (off-white) in the interface of the homology model for SARS-CoV-2; (B) Binding models of Cepharanthine (cyan) in the SARS-CoV-2 NSP12NSP8 protein interface pocket (white surface).

\subsection{Docking results of Filibuvir against SARS-CoV-2 NSP12-NSP8 model}

Filibuvir is an effective oral non-nucleoside HCV NS5B polymerase inhibitor for the potential treatment of chronic HCV infection [20]. Studies have shown that Filibuvir was well tolerated and could be considered in combination with other antiviral drugs to achieve better safety and efficacy for chronic HCV [21]. Our docking results showed that Filibuvir was mainly combined with the SARS-CoV-2 NSP12-NSP8 interface through van der Waals potential energy (Figure 6A). Filibuvir could bind to the interface active pockets of the SARS-CoV-2 NSP12-NSP8 (Figure 6B). Thus, Filibuvir can be considered as a candidate drug for treating SARS-CoV-2 infection, providing evidence for further research. 

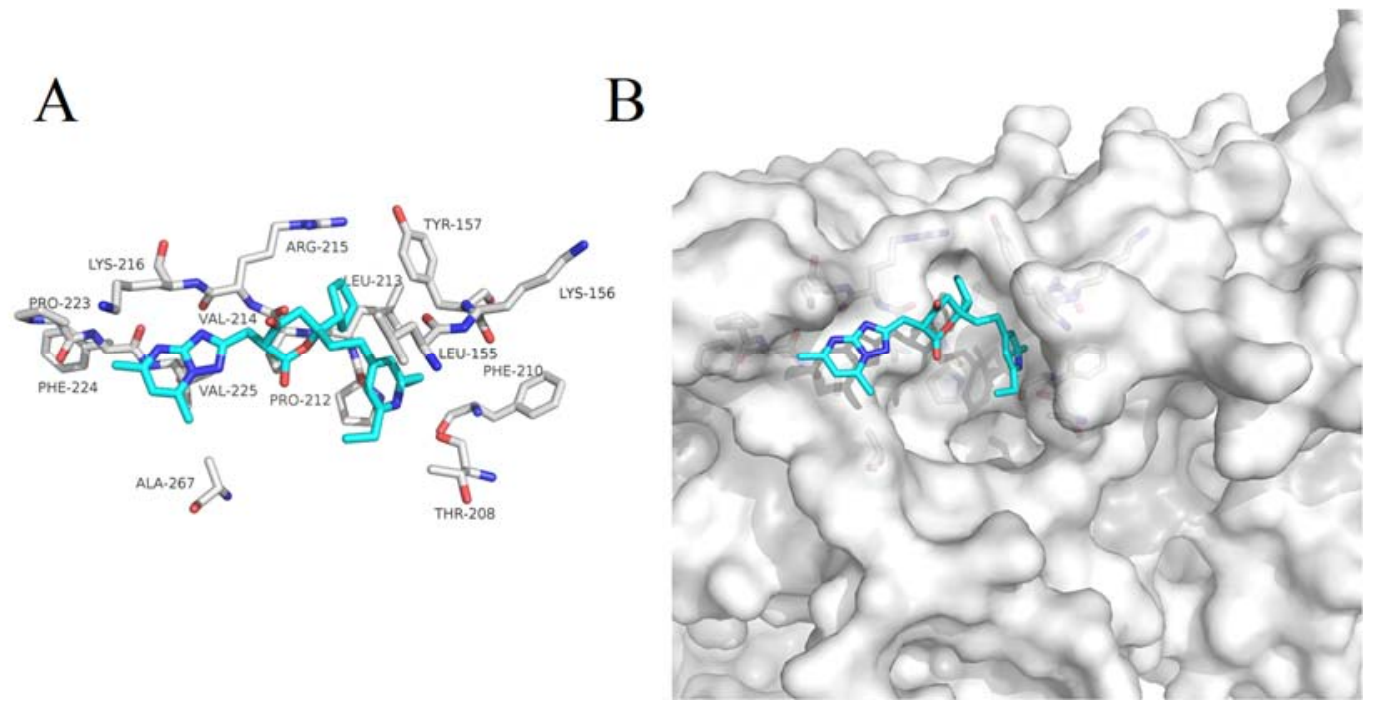

Figure 6. The binding model of Filibuvir against SARS-CoV-2 NSP12-NSP8. (A) Interactions between Filibuvir (cyan) and associated residues (off-white) in the interface of the homology model for SARS-CoV-2; (B) Binding models of Filibuvir (cyan) in the SARS-CoV-2 NSP12-NSP8 protein interface pocket (white surface).

\subsection{Binding Free Energy calculated by MM/GBSA}

Through the simulation trajectory of 5 ns molecular dynamics (MD) simulations, we calculated the binding free energy of 8 drugs by MM/GBSA method. The calculated binding free energies of Saquinavir, Tipranavir, Tegobuvir and Lonafarnib for the NSP12-NSP7 model were $-23.0269 \pm 4.4383,-12.1704 \pm 3.2929,-24.4461 \pm 3.5461$, $16.8649 \pm 1.8442 \mathrm{kcal} / \mathrm{mol}$ respectively, which highlighted Tegobuvir as the most active one (Table 3).The interaction of van der Waals forces contributed more than the electrostatic interaction for Tipranavir, Tegobuvir and Lonafarnib, indicating that van der Waals force is the main driving force for the combination of the three drugs. In addition, the calculated binding free energies of Olysio, Tegobuvir, Filibuvir and Cepharanthine for the NSP12-NSP8 model were -28.5431 $\pm 3.4974,-23.8177 \pm 2.8786$, $30.0087 \pm 2.6150,-27.9163 \pm 3.1346 \mathrm{kcal} / \mathrm{mol}$ respectively (Table 4), indicating Filibuvir with the strongest binding free energy. 
Table 3 -The calculated binding energies of ligand to the interface of SARS-CoV-2 NSP12-NSP7

Table 3-The calculated binding energies of ligand to the interface of NSP12-NSP7

\begin{tabular}{ccccc} 
Energy* & Saquinavir & Tipranavir & Lonafarnib & Tegobuvir \\
\hline$\Delta \mathrm{E}_{\mathrm{vdw}}$ & $-32.8851 \pm 4.4965$ & $-26.6226 \pm 4.9321$ & $-32.2453 \pm 4.5440$ & $-25.4293 \pm 2.1237$ \\
$\Delta \mathrm{E}_{\mathrm{ele}}$ & $-68.8922 \pm 8.2672$ & $-0.0371 \pm 2.7852$ & $-0.3949 \pm 3.4435$ & $-8.4345 \pm 2.4795$ \\
$\Delta \mathrm{G}_{\mathrm{gb}}$ & $82.9788 \pm 8.3596$ & $18.1213 \pm 3.9815$ & $11.6541 \pm 2.7204$ & $20.1027 \pm 2.4178$ \\
$\Delta \mathrm{G}_{\mathrm{np}}$ & $-4.2285 \pm 0.5503$ & $-3.6321 \pm 0.5170$ & $-3.4600 \pm 0.4934$ & $-3.1038 \pm 0.1822$ \\
$\Delta \mathrm{G}_{\mathrm{cal}}$ & $-23.0269 \pm 4.4383$ & $-12.1704 \pm 3.2929$ & $-24.4461 \pm 3.5461$ & $-16.8649 \pm 1.8442$ \\
\hline $\begin{array}{l}* \\
\mathrm{E}_{\mathrm{vdw}}=\end{array}$ van der Waals energy terms; $\Delta \mathrm{E}_{\mathrm{ele}}=\mathrm{electrostatic}$ energy; $\Delta \mathrm{G}_{\mathrm{gb}}=$ polar solvation free \\
energy; $\Delta \mathrm{G}_{\mathrm{np}}=$ nonpolar solvation free energy; $\Delta \mathrm{G}_{\mathrm{cal}}=$ final estimated binding free energy \\
calculated from the above terms $(\mathrm{kCal} / \mathrm{mol})$.
\end{tabular}

Table 4 -The calculated binding energies of ligand to the interface of SARS-CoV-2 NSP12-NSP8

\section{Table 4 -The calculated binding energies of ligand to the interface of NSP12-NSP8}

\begin{tabular}{ccccc} 
Energy* & Olysio & Tegobuvir & Filibuvir & Cepharanthine \\
\hline$\Delta \mathrm{E}_{\mathrm{vdw}}$ & $-48.0204 \pm 2.7890$ & $-33.3370 \pm 2.6790$ & $-41.9872 \pm 2.6531$ & $-39.1550 \pm 3.0045$ \\
$\Delta \mathrm{E}_{\mathrm{ele}}$ & $-1.3445 \pm 9.7221$ & $-14.0331 \pm 4.1102$ & $-11.8905 \pm 2.4355$ & $-164.7184 \pm 10.2539$ \\
$\Delta \mathrm{G}_{\mathrm{gb}}$ & $26.6238 \pm 7.9551$ & $28.1508 \pm 3.1191$ & $29.0768 \pm 2.5574$ & $180.0938 \pm 9.6691$ \\
$\Delta \mathrm{G}_{\mathrm{np}}$ & $-5.8020 \pm 0.3219$ & $-4.5984 \pm 0.2536$ & $-5.2077 \pm 0.2469$ & $-4.1367 \pm 0.2773$ \\
$\Delta \mathrm{G}_{\mathrm{cal}}$ & $-28.5431 \pm 3.4974$ & $-23.8177 \pm 2.8786$ & $-30.0087 \pm 2.6150$ & $-27.9163 \pm 3.1346$ \\
\hline
\end{tabular}

\section{Conclusions}

As of February 26, 2020, SARS-CoV-2 has caused 2,718 deaths in China, posing a threat to global human health. So far, no drug has been officially approved to treat SARS-CoV-2 infection. SARS-CoV-2 NSP12-NSP7-NSP8 complex is a potential target to fight against the virus. As for a highly active NSP12 polymerase complex, viral cofactors NSP7 and NSP8 are essential, suggesting that the particularly interesting compounds could disrupt the binding of NSP7 or NSP8 to NSP12. Thus, this study established two homologous models for the NSP12-NSP7-NSP8 complex, namely the NSP12-NSP7 interface model and the NSP12-NSP8 interface model. Subsequently, 24 compounds were selected for further evaluation based on virtual screening and docking scores, leading 7 compounds for the calculation of binding free energy. The seven 
compounds were Saquinavir, Tipranavir, Lonafarnib, Tegobuvir, Olysio, Filibuvir and Cepharanthine, respectively. According to published literature, all of them have antiviral activity. Therefore, this study suggests that seven compounds can be tested in vitro for their anti- SARS-CoV-2 effect, providing more choices for clinical treatment and prevention of SARS-CoV-2 infection.

\section{Acknowledgments}

This work was supported by grants from the National Natural Science Foundation of China (NO. 31870135, NO.31600116) and the "1000 Talent Plan" of Sichuan Province (NO. 980, NO.1060).

\section{References}

[1] P. Zhou, X.L. Yang, X.G. Wang, B. Hu, L. Zhang, W. Zhang, H.R. Si, Y. Zhu, B. Li, C.L. Huang, H.D. Chen, J. Chen, Y. Luo, H. Guo, R.D. Jiang, M.Q. Liu, Y. Chen, X.R. Shen, X. Wang, X.S. Zheng, K. Zhao, Q.J. Chen, F. Deng, L.L. Liu, B. Yan, F.X. Zhan, Y.Y. Wang, G.F. Xiao, Z.L. Shi, A pneumonia outbreak associated with a new coronavirus of probable bat origin, Nature (2020).

[2] H. P, S.E.J.T.i.j.o. occupational, e. medicine, The Novel Coronavirus: A Bird's Eye View, 11(2) (2020) 65-71.

[3] T.H.J.Z.y.f.y.x.z. zhi, [2019-nCoV: new challenges from coronavirus], 54 (2020) E001.

[4] Y. F, L. N, W. JY, H. LL, S. GS, Z.N.J.Z.j.h.h.h.X.z.z.Z.j.h.h.z.C.j.o. tuberculosis, r. diseases, [Pulmonary rehabilitation guidelines in the principle of $4 \mathrm{~S}$ for patients infected with 2019 novel coronavirus (2019-nCoV)], 43 (2020) E004.

[5] E.J. Snijder, E. Decroly, J. Ziebuhr, The Nonstructural Proteins Directing Coronavirus RNA Synthesis and Processing, Adv Virus Res 96 (2016) 59-126.

[6] A. Zumla, J.F. Chan, E.I. Azhar, D.S. Hui, K.Y. Yuen, Coronaviruses - drug discovery and therapeutic options, Nat Rev Drug Discov 15(5) (2016) 327-47.

[7] L. Subissi, C.C. Posthuma, A. Collet, J.C. Zevenhoven-Dobbe, A.E. Gorbalenya, E. Decroly, E.J. Snijder, B. Canard, I. Imbert, One severe acute respiratory syndrome coronavirus protein complex integrates processive RNA polymerase and exonuclease activities, Proc Natl Acad Sci U S A 111(37) (2014) E3900-9.

[8] R.N. Kirchdoerfer, A.B. Ward, Structure of the SARS-CoV nsp12 polymerase bound to nsp7 and nsp8 co-factors, Nat Commun 10(1) (2019) 2342.

[9] A.J. te Velthuis, S.H. van den Worm, E.J. Snijder, The SARS-coronavirus nsp7+nsp8 complex is a unique multimeric RNA polymerase capable of both de novo initiation and primer extension, Nucleic Acids Res 40(4) (2012) 1737-47.

[10] 1.P.C.J.E.o.o.d. metabolism, toxicology, Saquinavir, the pioneer antiretroviral protease inhibitor, 5(10) (2009) 1313-22. 
[11] K. VR, K. H, F.C.J.E.r.o.a.-i. therapy, Tipranavir: a novel second-generation nonpeptidic protease inhibitor, 3(1) (2005) 9-21.

[12] B.R. Beck, B. Shin, Y. Choi, S. Park, K. Kang, Predicting commercially available antiviral drugs that may act on the novel coronavirus (2019-nCoV), Wuhan, China through a drug-target interaction deep learning model, bioRxiv (2020).

[13] W. NS, M.M.J.E.o.o.i. drugs, Lonafarnib for cancer and progeria, 21(7) (2012) 1043-55.

[14] A. T, L. D, T. I, C. C, B. N, M. P, M.A.J.L.i.o.j.o.t.I.A.f.t.S.o.t. Liver, Future treatments for hepatitis delta virus infection, (2020) 54-60.

[15] V. I, P. J, Z. W, N.J.J.A. research, In vitro combinations containing Tegobuvir are highly efficient in curing cells from HCV replicon and in delaying/preventing the development of drug resistance, 120 (2015) 112-21.

[16] Z. S, B. P, A. K, M. P, S. D, K. H, M. C, Z. JP, H. Y, M. H, A. S, K. S, O. D, M. JG, M. MP, F.G.J. Hepatology, The protease inhibitor, GS-9256, and non-nucleoside polymerase inhibitor tegobuvir alone, with ribavirin, or pegylated interferon plus ribavirin in hepatitis C, 55(3) (2012) 749-58.

[17] T.K.J.A.j.o.h.-s.p.A.o.j.o.t.A.S.o.H.-S. Pharmacists, Simeprevir approved for hepatitis C virus infection, 71(1) (2014) 6.

[18] W. C, O.T.J.T.A. Chemistry, biology, Bisbenzylisoquinoline Alkaloids, 81 (2019) 1-114.

[19] L. X, W. Y, Z. M, L. G, C.Y.J.Z.y.c.Z.J.o.C.m. materials, [Study on the inhibitory effect of Cepharanthine on herpes simplex type-1 virus (HSV-1) in vitro], 27(2) (2004) 107-10.

[20] R.-T. M, Y. EM, M. P, S. S, P. VS, W. C, H.J.J.A.o. hepatology, A phase 2 study of filibuvir in combination with pegylated IFN alfa and ribavirin for chronic HCV, 13(4) (2014) 364-75.

[21] M. Rodriguez-Torres, E.M. Yoshida, P. Marcellin, S. Srinivasan, V.S. Purohit, C. Wang, J.L. Hammond, A phase 2 study of filibuvir in combination with pegylated IFN alfa and ribavirin for chronic HCV, Ann Hepatol 13(4) (2014) 364-75. 